\title{
Dominant Inheritance of Large Molecular Weight Immunoreactive Glucagon
}

\author{
Jerry P. Palmer, Phillip L. Werner, James W. Benson, and \\ John W. Ensinck, Department of Medicine and the Diabetes \\ Research Center, University of Washington, Seattle, \\ Washington 98144
}

\begin{abstract}
A B S T R A C T Plasma from some individuals contains substances which are reactive with glucagon antiserum, are larger than 3,500-dalton glucagon, and have been proposed as possible precursors of glucagon. We have evaluated three generations of a kindred in which 9 of 15 members evaluated had elevated plasma levels of large molecular weight immunoreactive glucagon (L-IRG) with an average concentration of $822 \mathrm{pg} / \mathrm{ml}$. The distribution of individuals with elevated L-IRG levels in this pedigree is consistent with autosomal dominant inheritance. Gel filtration of plasma revealed that all affected family members had excessive amounts of two L-IRG peaks, one with a molecular weight of approximately 9,000 daltons and another in the 10,000 to 20,000 -dalton range. Oral glucose tolerance tests were nondiabetic and elicited a fall in L-IRG levels, whereas L-IRG concentrations rose dramatically during the infusion of arginine. These L-IRG species may be precursors of 3,500-dalton glucagon and may be elevated in this kindred because of an inherited defect in either their synthesis or degradation.
\end{abstract}

\section{INTRODUCTION}

Human plasma contains more than one species of immunoreactive somatotropin, gastrin, parathyroid

This work was presented in part at the 36th Annual Meeting of the American Diabetes Association and at the International Symposium on Glucagon and published as an abstract (1976. Diabetes. 25[Suppl. 1]: 326) and a preliminary report (1976. Metab. Clin. Exp. 25[Suppl. 1]: 1483) in connection with these meetings, respectively.

Dr. Werner was the recipient of a fellowship award from the American Diabetes Association, Washington Affiliate. His present address is Emory University School of Medicine, Grady Hospital, Diabetes Unit, Atlanta, Ga. 30303. Dr Benson's present address is The Mason Clinic, Seattle, Wash. 98101.

Received for publication 15 April 1977 and in revised form 4 November 1977. hormone, insulin, and glucagon which have been distinguished by differences in molecular weight $(1,2)$. The precise biologic significance of many of these compounds is not fully understood, whereas some, such as proinsulin, are known to be biosynthetic precursors of the smaller molecular weight, biologically more active species. Plasma levels of proinsulin increase after glucose administration and are elevated in patients with several clinical conditions, most notable being patients with insulinomas (3). Recently, Gabbay et al. have described a family with hyperproinsulinemia inherited in an autosomal dominant pattern (4). Pancreatic vein catheterization has revealed that the majority of the immunoreactive glucagon (IRG) ${ }^{1}$ secreted by pancreatic alpha cells in the dog has a molecular weight of approximately 3,500 daltons (5). Species of IRG larger and smaller than 3,500-dalton glucagon have been described in human plasma (6-8). Noe and Bauer have found a polypeptide of approximately 9,000 daltons which, by pulse-chase experiment, appears to be an intermediate in glucagon biosynthesis (9). Therefore, the 9,000-dalton IRG species observed in human plasma may represent a precursor form of 3,500-dalton glucagon.

We have evaluated a family with excessive plasma levels of large molecular weight IRG (L-IRG) but normal levels of 3,500-dalton IRG, in which the pattern in the pedigree is consistent with autosomal dominant inheritance. The L-IRG in affected members consists of species with mol wt 9,000 and approximately 10,000-20,000 daltons, which are similar to the molecular weight of the putative precursors of 3,500-dalton glucagon which have been observed in human and animal in vitro pancreas studies $(9,10)$. In addition, plasma levels of these L-IRG species

\footnotetext{
${ }^{1}$ Abbreviations used in this paper: IRG, immunoreactive glucagon; L-IRG, large molecular weight immunoreactive glucagon; S-IRG, small molecular weight immunoreactive glucagon.
} 
were decreased during hyperglycemia and elevated by arginine, implying that they may be secretory products of alpha cells. This kindred may represent familial hyperproglucagonemia.

\section{METHODS}

15 individuals in three generations of this kindred, ranging in age from 8 to $71 \mathrm{yr}$ old, were evaluated. All were healthy, had weights ranging from 100 to $148 \%$ of ideal (11), and had no personal or family history of diabetes or endocrine neoplasia. None had known renal impairment. Subjects were informed of the nature, purpose, and potential risks before their voluntary participation. Blood was withdrawn and arginine injected by scalp vein needles in the subjects' antecubital veins. Patency of these lines was maintained by the slow infusion of normal saline. The oral glucose tolerance test $(100 \mathrm{~g})$, arginine infusions $(500 \mathrm{mg} / \mathrm{kg}$ per $30 \mathrm{~min}$ ), and arginine pulses $(5 \mathrm{~g} / 30 \mathrm{~s}$ ) were performed after an overnight fast with a 3-day high carbohydrate diet preceding the oral glucose tolerance test.

Each plasma sample was assayed for IRG (30K) level in both the native state and after treatment with acetone. The 3,500 -dalton glucagon is soluble in acetone with $75 \%$ recovery (12). The 160,000 -dalton species is virtually excluded by precipitation, and we have found that less than $10 \%$ of the 9,000-dalton and 10,000- to 20,000-dalton species is extracted into acetone. Therefore, the IRG level in the acetone extract of plasma represents primarily the smaller molecular weight glucagon, which we have designated S-IRG. Since IRG in native plasma measures all IRG species, the difference between IRG in unextracted plasma and S-IRG allows quantification of the larger molecular weight glucagon species. Glucose was measured by an AutoAnalyzer adapta- tion of the ferrocyanide or neocuproine reduction method and insulin by radioimmunoassay (13). 1-ml samples of plasma were fractionated on a $1.5 \times 90-\mathrm{cm}$ Bio-Gel P-10 column (BioRad Laboratories, Richmond, Calif.) in $0.05 \mathrm{M}$ Veronal buffer plus $0.25 \%$ bovine serum albumin at $\mathrm{pH} 8.6$. The columns were standardized by applying proinsulin, insulin, and glucagon to the columns and measuring by radioimmunoassay the eluates for these substances. 400- $\mu \mathrm{l}$ aliquots of the $1-\mathrm{ml}$ fractions were assayed for IRG using antiserum $30 \mathrm{~K}$. Recoveries were calculated as the sum of the IRG measured in the eluate fractions divided by the total IRG in the respective native plasma.

\section{RESULTS}

The pedigree of this family is shown in Fig. 1. All affected members of this kindred had S-IRG levels within our normal range, which was defined as the mean fasting $S-I R G \pm 2 S D$ in 23 nondiabetic control subjects $(21-101 \mathrm{pg} / \mathrm{ml})$. The mean fasting level of $\mathrm{L}$ IRG plus $2 \mathrm{SD}(62+70=132 \mathrm{pg} / \mathrm{ml})$ of the 23 nondiabetic control volunteers was taken as the upper limit of normal; therefore family members with L-IRG values exceeding $132 \mathrm{pg} / \mathrm{ml}$ were considered affected. Plasma for L-IRG and S-IRG of affected individuals was obtained after an overnight fast except for J. S., whose specimen was drawn at random. Of the 15 individuals evaluated, 9 were affected with L-IRG values ranging from 246 to $3,618 \mathrm{pg} / \mathrm{ml}$ (mean \pm SD $=822 \pm 1,059$ ). Five blood relatives of the propositus had normal L-IRG levels. 3 of the 15 family members

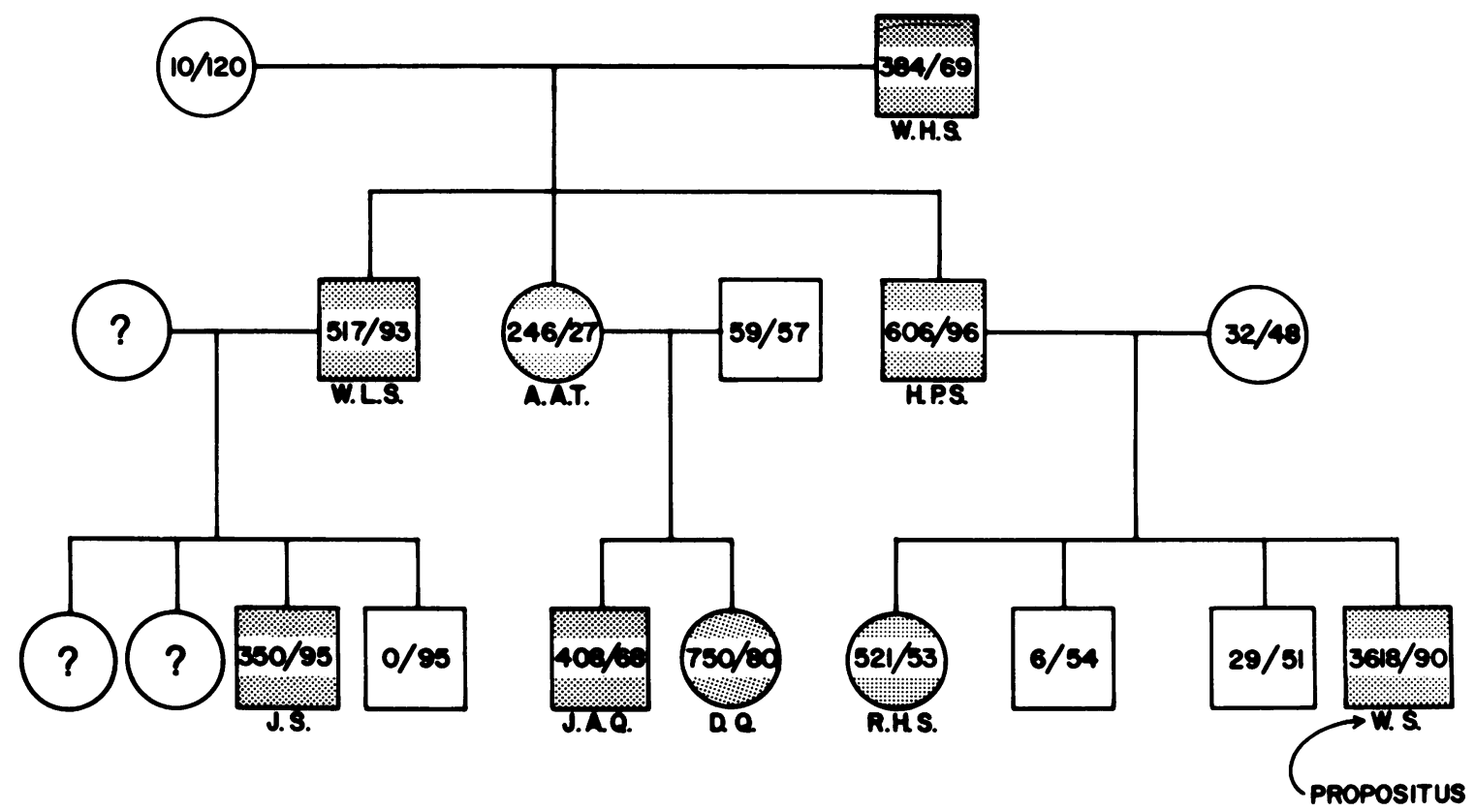

FIGURE 1 Inheritance pattern of excessive large molecular weight IRG in three generations of a family. Affected individuals are shaded, nonaffected are clear, and a question mark (?) represents individuals not tested. Numbers represent L-IRG/S-IRG. Letters below each box represent the individual's initials. 
were relatives by marriage, had normal L-IRG levels, and thereby served as internal controls. Individuals in all three generations evaluated and both men and women were affected.

Fig. 2 illustrates the gel-filtration profile of plasma IRG from J. A. Q. and depicts the pattern which characterizes this family. All affected members had excessive levels of L-IRG distributed in two peaks: one with a molecular weight similar to proinsulin, 9,000 daltons (peak A), and another slightly larger weight species eluting earlier from the Bio-Gel P-10 column (peak B). We have estimated the molecular weight of peak $B$ to be approximately $10,000-20,000$ daltons because of its position between the void volume of the column (20,000 daltons) and the proinsulin marker. Some but not all affected subjects had a peak which was excluded from the gel (peak C). By gel filtration on Sephadex G-100 and G-200 columns (Pharmacia Fine Chemicals, Inc., Piscataway, ‥ J.), we and others have found this peak of IRG to have a molecular weight of about 160,000 daltons $(6,7)$. There was considerable variability between subjects, with some individuals having no detectable void volume peak (W. H. S., W. L. S., H. P. S.), while in other subjects it comprised $48 \%$ and $62 \%$ of the total IRG (R. H. S. and W. S., respectively). Similarly; there was a wide range for peak $\mathrm{A}(11-72 \%)$ and for peak B (18-76\%). Because of the limits of assay sensitivity we did not always detect a distinct peak in the area corresponding to 3,500-dalton IRG.

Six affected family members (W. H. S., W. L S., H. P. S., J. A. Q., W. S., R. H. S.) had oral glucose tolerance tests which were all nondiabetic (Fig. 3). Mean S-IRG concentrations fell from a fasting level of $80 \mathrm{pg} / \mathrm{ml}$ to a nadir at $3 \mathrm{~h}$ of $59 \mathrm{pg} / \mathrm{ml}$. Mean L-IRG levels decreased $200 \mathrm{pg} / \mathrm{ml}$ during the oral glucose tolerance test, and when each individual's values were compared with his (her) fasting level, the decrements in levels were significant $(P<0.05)$ at each hour after administration of the glucose. Basal IRI and the IRI

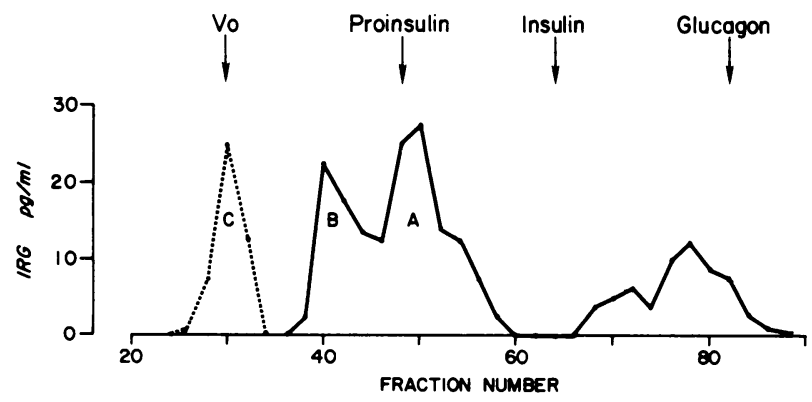

FIGURE 2 IRG concentrations in eluates from gel filtration on Bio-Gel P-10 of J. A. Q.'s plasma (recovery $=100 \%$ ). Peaks A and $B$ (solid line) were observed in all affected family members, whereas peak $C$, (dashed line) was observed in only some members.
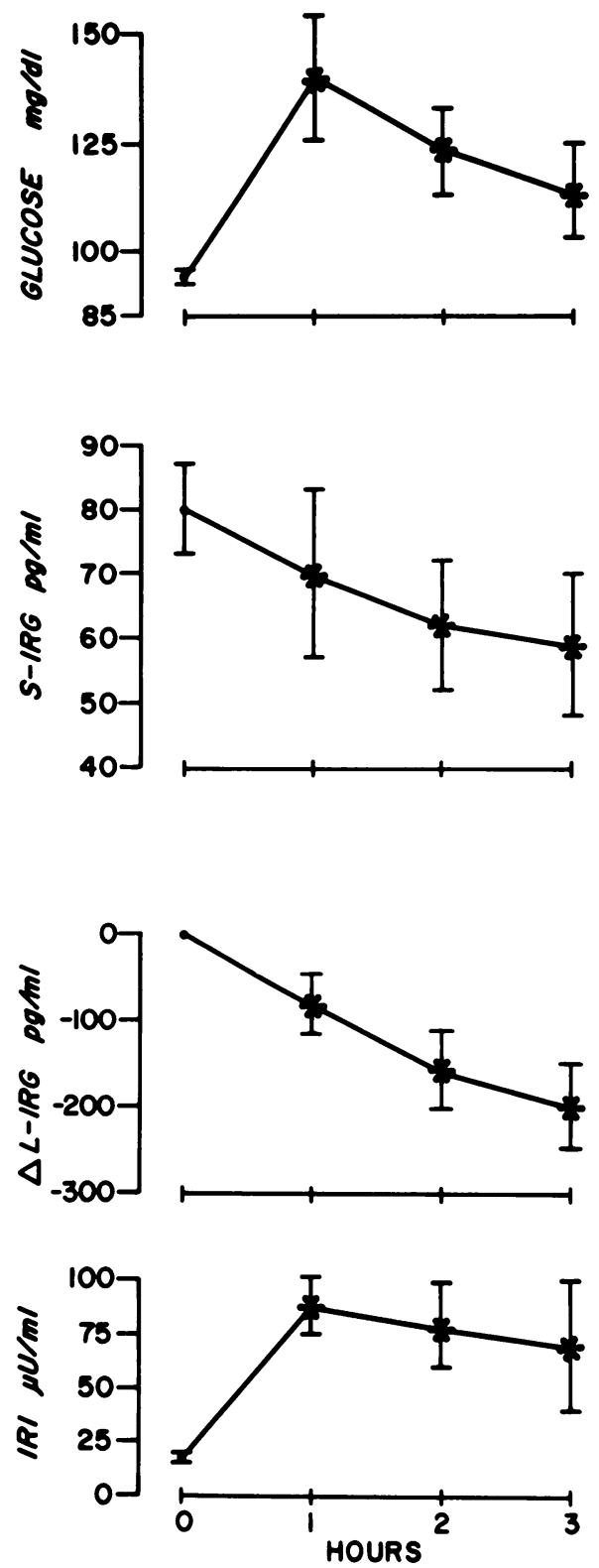

Figure 3 Oral glucose tolerance tests $(100 \mathrm{~g})$ in six affected family members. Values are mean \pm SEM except for L-IRG graph, which is depicted as change from basal. An asterisk (*) denotes values significantly $(P<0.05)$ different from fasting value by paired comparison analysis.

responses to the $100 \mathrm{~g}$ of glucose were normal when compared with values obtained by others (14).

The elution profiles of L-IRG from W. L. S. in the fasting state and 3-h after oral glucose are shown in Fig. 4. Both peaks $A$ and $B$ were depressed after the administration of glucose.

The S-IRG and L-IRG responses to the arginine infusion in four affected family members (H. P. S., J. A. Q., W. H. S., R. H. S.) and in seven non- 


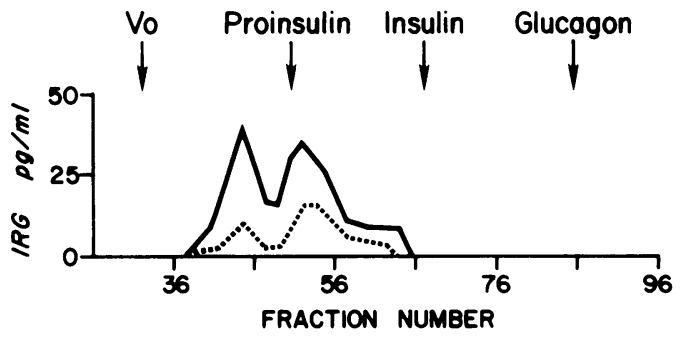

FIGURE 4 IRG concentrations in eluates (Bio-Gel P-10) from plasma of W. L. S. fasting (solid line) and $3 \mathrm{~h}$ after $100 \mathrm{~g}$ of oral glucose load (dashed line). Fasting recovery $=65 \% 3-\mathrm{h}$ recovery $=70 \%$.

diabetic controls are shown in Fig. 5. L-IRG in the control volunteers rose only slightly during the arginine infusion, achieving significant $(P<0.05)$ elevation above basal at intermittent time points (see
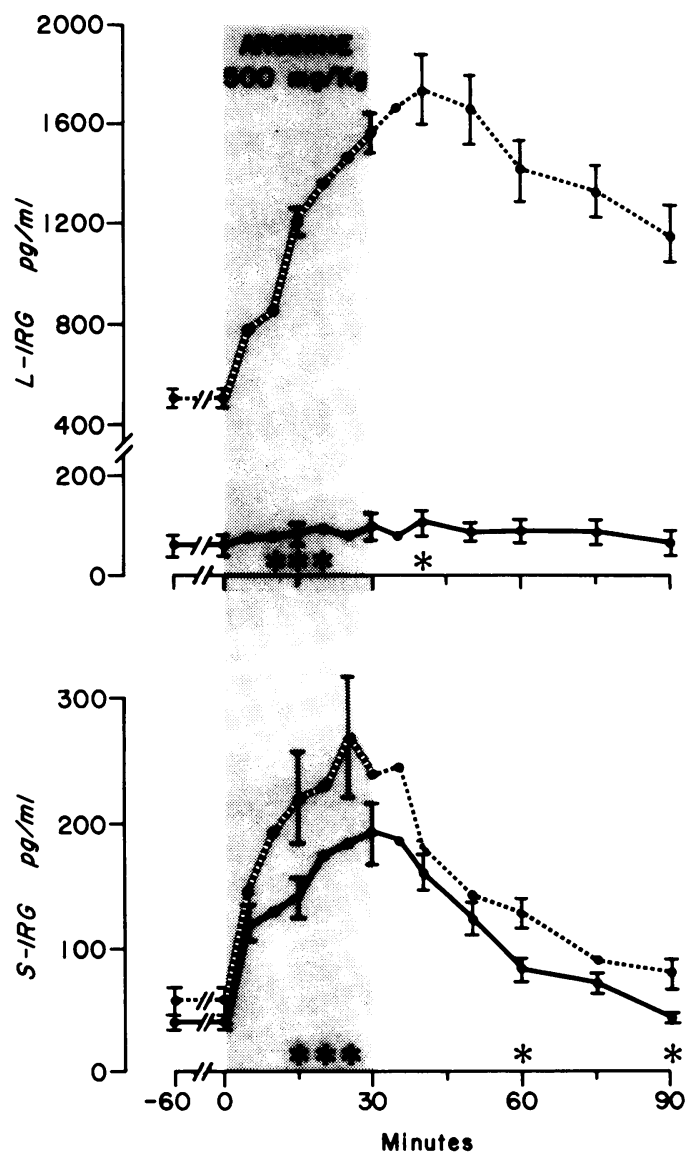

FIGURE 5 L-IRG and S-IRG levels in seven controls (solid line) and four affected family members (dashed line) during an arginine infusion. The asterisk $(*)$ in the L-IRG graph designates values significantly different $(P<0.05)$ from fasting values in the control subjects. The astrisk $(*)$ in the S-IRG graph indicates times when S-IRG in family members was significantly different $(P<0.05)$ from S-IRG values in control subjects.

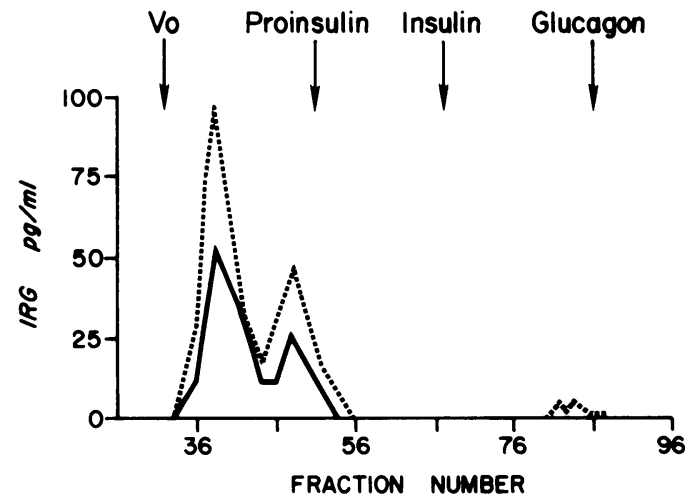

FIGURE 6 IRG concentrations in eluates (Bio-Gel P-10) from plasma of H. P. S. fasting (solid line) and $90 \mathrm{~min}$ after arginine stimulation (dashed line). Fasting recovery $=45 \%$; postarginine recovery $=51 \%$.

Fig. 5). In contrast, the family members demonstrated marked increases of L-IRG concentrations with mean levels increasing from $506 \mathrm{pg} / \mathrm{ml}$ to a maximum of $1,727 \mathrm{pg} / \mathrm{ml}$ and then slowly declining. S-IRG rose briskly in both controls and family members and fell promptly after infusion to near basal values by 90 min. Mean S-IRG levels during the arginine infusion were slightly higher in the family members than in the control subjects but achieved statistical significance only intermittently. The gel filtration profile of plasma IRG from $H$. P. S. in the fasting state and at $90 \mathrm{~min}$ of the arginine infusion is shown in Fig. 6. Both peaks $\mathrm{A}$ and $\mathrm{B}$ were increased, and a small peak corresponding to the 3,500-dalton glucagon marker was observed after arginine. The bolus administration of arginine in W. H. S., H. P. S., and D. Q. resulted in acute elevation of both S-IRG and L-IRG levels. At 2 min after arginine administration, mean S-IRG and L-IRG concentrations had risen 98 and $226 \mathrm{pg} / \mathrm{ml}$, respectively. Serum creatinine levels were measured in J. A. Q., D. Q., W. H. S., R. H. S., and W. S., and all were normal.

\section{DISCUSSION}

The amino acid sequence of proinsulin, the specific sites of cleavage of proinsulin to insulin, and therefore the definite precursor-product relationship of proinsulin and insulin have been clearly established (15). Glucagon may also be synthesized initially as a larger polypeptide with one or more cleavage steps being required to elaborate 3,500-dalton glucagon. Studies in fish, birds, and mammals have identified an array of larger molecular weight IRG species and tryptophanlabeled islet secretory products which have been considered as possible proglucagon(s) $(1,9,10,16-21)$. Some of this apparent heterogeneity may be due to species variability. Pulse-chase experiments by Noe 
and Bauer with $\left[{ }^{3} \mathrm{H}\right]$ tryptophan in anglerfish islets have provided evidence for sequential cleavage of 11,400-dalton proglucagon to a 9,000-dalton conversion intermediate and subsequently to 3,500)-dalton glucagon (9). In marked contrast, O'Connor and Lazarus found no precursor-product relationship between 9,000- and 3,500-dalton glucagon in pigeon islets (21). In addition to 3,500-dalton IRG, human plasma contains variable amounts of 160,000- and 9,000dalton IRG, with elevated levels of the latter being common in patients with chronic renal failure $(8,22)$ and in those with glucagonomas (23-26). Studies with human islets have demonstrated the incorporation of $\left[{ }^{3} \mathrm{H}\right]$ tryptophan into both 9,000 - and 3,500dalton IRG (10). In the pedigree reported here, all affected family members had excessive levels of L-IRG with molecular weights of 9,000 and $10,000-20,000$ daltons. IRG of the latter molecular weights has not been previously described in human plasma, but it may correspond to the 11,400 IRG species found by Noe and Bauer in the anglerfish (9). Our observations that the plasma levels of both the L-IRG species that characterize this family rise and fall appropriately with arginine and glucose administration, respectively, and especially the finding that L-IRG is elevated 2 min after an arginine pulse, suggest that these LIRG species may be alpha cell secretory products. None of the family members had evidence of renal impairment by history, and in five affected family members serum creatinine levels were found to be normal. Recently, Gabbay et al. have reported the autosomal dominant inheritance of familial hyperproinsulinemia (4). Although we have no direct evidence that the excessive L-IRG species which characterize this pedigree are glucagon precursors, the autosomal dominant inheritance pattern of elevated L-IRG levels, the similarity in molecular weights of this kindred's L-IRG to species which are candidates for proglucagon(s), and the L-IRG responses to stimulation and suppression that we observed all provide support for this hypothesis.

Bio-Gel P-10, Bio-Gel P-30, and Sephadex have been used for the chromatographic separation of the different molecular weight species of IRG. The columns we employed are essentially the same as those reported by Noe and Bauer $(1.6 \times 90+\mathrm{cm}$ Bio-Gel P-10) to give enhanced separation (9). In preliminary studies comparing Bio-Gel P-10 and P-30, we observed superior separation of affected family members' plasma L-IRG components with Bio-Gel P-10. Bio-Gel P-30 chromatography of plasma from H. P. S. and W. L. S. by J. B. Jaspan revealed an IRG peak eluting in the region of the proinsulin marker and another peak between the gamma globulin marker and the proinsulin marker. The appearance of the latter peak, well to the right of the gamma globulin marker, suggests that this IRG peak has a molecular weight intermediate between the commonly observed void volume IRG and the 9,000 IRG. ${ }^{2}$

Patients with insulinomas frequently have high levels of proinsulin (3), and therefore the question arises whether the high L-IRG levels could be indicative of alpha cell tumors in our subjects. Patients with glucagonomas frequently have a severe skin rash and carbohydrate intolerance $(23,24)$; none of the affected family members had dermatitis and all had normal glucose tolerance tests. Although excessive levels of large molecular weight IRG have been reported in glucagonoma patients, these patients also had excessive levels of 3,500-dalton IRG $(25,26)$. The levels of S-IRG, which represent predominantly 3,500 IRG, were normal in each of our subjects (Fig. 1), thereby providing further evidence that they did not have islet cell neoplasms.

Boden and Owen have described hyperglucagonemia in four relatives of a glucagonoma patient. Noninvasive techniques failed to show any evidence for pancreatic neoplasm in these relatives. Gel filtration on Sephadex G-50 fine revealed excessive levels of IRG eluting between the proinsulin marker and the void volume and within the void volume (27). It is possible that the large molecular weight IRG peak in these subjects contains both the 9,000dalton and the 10,000- to 20,000-dalton IRG peaks characteristic of our family.

As shown in fig. 5, S-IRG levels declined much more rapidly than L-IRG concentrations after termination of the arginine infusion. This would suggest a much slower metabolic clearance rate for the larger glucagon species and is analogous to the half-time differences reported for "big" versus "little" gastrin (28) and proinsulin versus insulin (29). Our data suggesting that L-IRG has a longer half-time of disappearance than S-IRG agrees qualitatively with the longer half-time of "big IRG" $(8,200$ daltons) than of "little IRG" (3,500 daltons) obtained by O'Connor and Lazarus in the rat (30). The slightly elevated mean SIRG levels of affected family members during the arginine infusion may be due to increased alpha cell responsiveness to arginine. Alternatively, the tendency for higher S-IRG levels may be methodologic. The excessive L-IRG response to arginine in the affected family members might result in spuriously high S-IRG levels in these individuals, since up to $10 \%$ of L-IRG may be extracted into the acetone and would then be measured as S-IRG.

Assan and Slusher have demonstrated that distinct and separate portions of the glucagon molecule account for its different biologic properties; the 19-22 amino acid sequence being responsible for lipolysis,

\footnotetext{
${ }^{2}$ Jaspan, J. B. Personal communication.
} 
whereas the structure necessary for the glycogenolytic and insulinogenic actions is located in the 24-29 amino acid sequence (31). Rigopoulou et al. reported that "big glucagon" was devoid of glycogenolytic activity in the perfused rat liver (32). O'Connor and Lazarus found no hyperglycemic effect upon injecting "big glucagon" ( 8,200 daltons) into rats, even though "big glucagon" did have lipolytic activity quantitatively comparable to 3,500-dalton glucagon (30). In contrast, other investigators have found little to no lipolytic activity for the larger molecular weight glucagon species (17), and very recently Srikant et al. have reported that 65,000-dalton IRG has glycogenolytic activity whereas the 9,000-dalton species does not (33). Perhaps some of the apparently conflicting results are a reflection of different separation procedures which may enable discrimination of two or more IRG species by one technique but failure to separate them when another method is employed. Although the relative concentrations of the different molecular weight IRG species that make up L-IRG in the subjects in our kindred are highly variable, the observation that, even with the very high L-IRG levels, glucose tolerance and insulin secretion are normal in affected family members strongly suggests that these L-IRG species have very little if any glycogenolytic or insulinogenic activity in our patients.

In summary, the kindred reported fulfilled the criteria of autosomal dominant inheritance of excessive levels of IRG species larger than 29 amino acid glucagon. The inherited defect may reside in the glucagon biosynthetic pathway with the excessive LIRG resulting from precursor buildup. Alternatively, the defect may be in the degradative system for the L-IRG species with consequent decreased ability to clear these IRG moieties from the blood. This mechanism probably accounts for the excessive 9,000-dalton IRG observed in the plasma of patients with chronic renal failure $(8,22)$. If these L-IRG molecules are precursors to 3,500-dalton IRG, this family illustrates the inheritance of hyperproglucagonemia.

\section{ACKNOWLEDGMENTS}

We are grateful to Ms. Claudine Nist, Ms. Ellen Laschansky, and Ms. Martha Knoeber for their excellent technical assistance; to Ms. Sharon Kemp and Ms. Pat Johnson for typing the manuscript; and to the family members for volunteering for these studies.

A portion of this work was conducted through the Clinical Research Center of the University of Washington, supported by National Institutes of Health grant RR-37. This investigation was supported in part by U. S. Public Health Service grants AM 16008-04 and SEA 76-44, a grant from the Research Foundation of the American Diabetes Association, and a grant from the American Diabetes Association (Washington Affiliate).

\section{REFERENCES}

1. Melani, F. 1974. Pro-hormones in tissues and in circulation. Horm. Metab. Res. 6: 1-8.

2. Yalow, R. S. 1974. Complexity of immunoreactive forms of peptide hormones in plasma and in tissue extracts. Isr. J. Med. Sci. 10: 1185-1200.

3. Gutman, R. A., N. R. Lazarus, J. C. Penhos, S. Fajans, and L. Recant. 1976. Circulating proinsulin-like material in patients with functioning insulinomas. N. Engl. J. Med. 281: 1003-1008.

4. Gabbay, K. H., K. DeLuca, J. N. Fisher, M. E. Mako, and A. H. Rubenstein. 1976. Familial hyperproinsulinemia. An autosomal dominant defect. N. Engl.J. Med. 294: $911-915$.

5. Valverde, I., D. Rigopoulou, D. B. Marco, G. R. Faloon, and R. Unger. 1970. Molecular size of extractable glucagon and glucagon-like immunoreactive (GLI) in plasma. Diabetes. 19: 624-629.

6. Weir, G. C., S. D. Knowlton, and D. B. Martin. 1975. High molecular weight glucagon-like immunoreactivity in plasma. J. Clin. Endocrinol. Metab. 40: 296-3()2.

7. Valverde I., M. L. Villanueva, I. Lozano, and L. Marco. 1974. Presence of glucagon immunoreactivity in the globulin fraction of human plasma ("big plasma glucagon”). J. Clin. Endocrinol. Metab. 39: 1090-1098.

8. Kuku S. F., A. Zeidler, D. S. Emmanouel, A. I. Katz, and A. H. Rubenstein. 1976. Heterogeneity of plasma glucagon: patterns in patients with chronic renal failure and diabetes. J. Clin. Endocrinol. Metab. 42: 173-176.

9. Noe, B. D., and G. E. Bauer. 1975. Evidence for sequential metabolic cleavage of proglucagon to glucagon in glucagon biosynthesis. Endocrinology 97: 868-877.

10. Noe, B. D., G. E. Bauer, M. W. Steffes, D. E. R. Sutherland, and J. S. Najarian. 1975. Glucagon biosynthesis in human pancreatic islets: preliminary evidence for a biosynthetic intermediate. Horm. Metab. Res. 7: 314-322.

11. Metropolitan Life Insurance Company Statistical Bulletin. 1959. New weight standards for men and women. 40: $1-11$.

12. Walter, R. M., J. Dudl, J. P. Palmer, and J. W. Ensinck. 1974. The effect of adrenergic blockade on the glucagon responses to starvation and hypoglycemia in man. $J$. Clin. Invest. 54: 1214-1220.

13. Morgan, C. R., and A. Lazerow. 1963. Immunoassay of insulin: two antibody systems. Plasma insulin levels of normal, subdiabetic and diabetic rats. Diabetes. 12: 115126.

14. Bagdade, J. D., E. L. Bierman, and D. Porte, Jr. 1967. The significance of basal insulin levels in the evaluation of the insulin response to glucose in diabetic and nondiabetic subjects. J. Clin. Invest. 46: 1549-1557.

15. Steiner, D. F. 1976. Errors in insulin biosynthesis. $N$. Engl. J. Med. 294: 952-953.

16. Peterson, K. G., P. Heilmeyer, and L. Kerp. 1975. Synthesis of proinsulin and large glucagon immunoreactivity in isolated Langerhans islets from EMC-virus infected mice. Diabetologia. 11: 21-25.

17. Hellerstrom, C., S. L. Howell, J. C. Edwards, A. Andersson, and C. Ostenson. 1974. Biosynthesis of glucagon in isolated pancreatic islets of guinea pigs. Biochem. J. 140: $13-23$.

18. Tager, H. S., and D. F. Steiner. 1973. Isolation of a glucagon-containing peptide: primary structure of a possible fragment of proglucagon. Proc. Natl. Acad. Sci. U. S. A. 70: 2321-2325. 
19. Tung, A. K. 1973. Biosynthesis of avian glucagon: evidence for a possible high molecular weight biosynthetic intermediate. Horm. Metab. Res. 5: 416-424.

20. Trakatellis, A. C., K. Tada, K. Yamaji, and P. GardikiKouindou. 1975. Isolation and partial characterization of anglerfish proglucagon. Biochemistry. 14: 1508-1512.

21. O'Connor, K. J., and N. R. Lazarus. 1976. Studies on the biosynthesis of pancreatic glucagon in the pigeon. Biochem. J. 156: 279-288.

22. Kuku, S. F., J. B. Jaspan, D. S. Emmanouel, A. Ziedler, A. I. Katz, and A. H. Rubenstein. 1976. Heterogeneity of plasma glucagon. Circulating components in normal subjects and patients with chronic renal failure. $J$. Clin. Invest. 58: 742-750.

23. McGavran, M. H., R. H. Unger, L. Recant, H. C. Polk, C. Kilo, and M. E. Levin. 1966. A glucagon-secreting alpha-cell carcinoma of the pancreas. N. Engl. J. Med. 274: 1408-1413.

24. Mallinson, C. N., S. R. Bloom, A. P. Warin, P. R. Salmon, and B. Cox. 1974. A glucagonoma syndrome. Lancet. II: 1-5.

25. Danforth, D. N., T. Triche, J. L. Doppman, R. M. Beazley, P. V. Perrino, and L. Recant. 1976. Elevated plasma proglucagon-like component with a glucagon-secreting tumor. N. Engl. J. Med. 295: 242-245.
26. Valverde, I., H. M. Lemon, A. Kessinger, and R. H. Unger. 1976. Distribution of plasma glucagon immunoreactivity in a patient with suspected glucagonoma. J. Clin. Endocrinol. Metab. 42: 804-808.

27. Boden, G., and O. E. Owen. 1977. Familial hyperglucagonemia - an autosomal disorder. N. Engl. J. Med. 296: $534-538$.

28. Straus, E., and R. S. Yalow. 1974. Studies on the distribution and degradation of heptadecapeptide, big, and big big gastrin. Gastroenterology. 66: 936-943.

29. Starr, J. I., and A. H. Rubenstein. 1974. Metabolism of endogenous proinsulin and insulin in man. J. Clin. Endocrinol. Metab. 38: 305-308.

30. O'Connor, K. J., and M. R. Lazarus. 1975. The purification and biological properties of pancreatic big glucagon. Biochem. J. 156: 265-277.

31. Assan, R., and N. Slusher. 1972. Structure/function and structure/immunoreactivity relationships of the glucagon molecule and related synthetic peptides. Diabetes. 21: 843-855.

32. Rigopoulou, D., I. Valverde, J. Marco, G. Faloona, and R. H. Unger. 1970. Large glucagon immunoreactivity in extracts of pancreas. J. Biol. Chem. 245: 496-501.

33. Srikant, C. B., K. McCorkle, and R. E. Dobbs. 1976. A biologically active "macro-'glucagon' (IRG)" in glucagonsecreting tissues of the dog. Diabetes. 25(Suppl. 1): 326. 